\title{
Vocal Fold Paresis: An Evolving Clinical Concept
}

\author{
Lucian Sulica
}

Published online: 8 May 2013

(C) Springer Science+Business Media New York 2013

\begin{abstract}
Neurogenic compromise of vocal fold function exists along a continuum encompassing variable degrees and patterns of partial denervation (paresis). Not abundantly recognized until recently, vocal fold paresis is probably a significant source of vocal disability, especially among cases that have eluded straightforward diagnosis. It presents with symptoms of glottic insufficiency, and probably follows a clinical course analogous to that of paralysis. As a result of preserved gross mobility, paresis can be difficult to diagnose and distinguish from innocent asymmetries of laryngeal motion. Both laryngoscopy/ stroboscopy and electromyography are useful in diagnosis despite both of them having important limitations. Because of these, trials of treatment are uniquely useful in vocal fold paresis. Significant disagreement persists regarding incidence, causes, signs, and association with other diseases. Treatment consists of medialization and/or augmentation procedures that do not compromise remaining neural function.
\end{abstract}

Keywords Larynx - Vocal fold · Paresis - Paralysis . Laryngeal neuropathy $\cdot$ Vagal neuropathy

\section{Introduction}

For most of the twentieth century, otolaryngologists conceptualized vocal fold paralysis as an all-or-none phenomenon, with paralysis-or, more precisely, absence of

\section{Sulica $(\square)$}

Department of Otolaryngology-Head and Neck Surgery,

Weill Cornell Medical College, 1305 York Avenue,

5th Floor, New York, NY 10021, USA

e-mail: lusulica@gmail.com motion-the product of a complete lack of neural input. Electrophysiologic investigations have shown that vocal fold paralysis is in fact a surprisingly heterogeneous clinical condition $[1,2 \cdot, 3]$. It is probably best considered as a continuum of neurogenic dysfunction, with the position of the vocal fold determined by a complex interaction between the degree of initial denervation and the degree and pattern of reinnervation. Given this potential for variability in nerve damage, it should come as no surprise that paresis, or partial paralysis in which some gross vocal fold mobility is preserved, exists as a clinical entity.

Although the existence of vocal fold paresis is beyond doubt, its incidence, presentation, and clinical significance remain under discussion. At the core of the debate is diagnosis, typically made on the basis of the observation of asymmetries of laryngeal motion. Distinguishing significant asymmetries from innocent findings may represent the greatest challenge in paresis, and electrophysiologic testing may not be as reliable a tool as is generally believed.

\section{Epidemiology}

Causes

Like paralysis, paresis appears to result from trauma to one or more laryngeal nerves, a variety of medical conditions, or damage from yet to be identified factors, designated "idiopathic." Malignant disease, an important category in the causes of paralysis, is notably rare, perhaps because its inherently progressive nature results in the rapid evolution of any paresis to paralysis before the patient comes to medical attention. A study of 60 patients has shown that imaging has no yield in cases of paresis [4]. The causes identified in the two series of paresis that have appeared in 
Table 1 Causes of vocal fold paresis (\%)

\begin{tabular}{lcl}
\hline & Koufman et al. & Heman-Ackah and \\
& {$[15 \bullet \bullet](n=50)$} & Barr [14・• $](n=19)$ \\
\hline Idiopathic or postviral & 68 & 42 \\
Malignancy & 6 & - \\
Postchemotherapy & 2 & - \\
$\quad$ neuropathy & & \\
Multiple sclerosis & 4 & - \\
Goiter/thyroiditis & - & 37 \\
Lyme disease & - & 5 \\
Intubation & 14 & - \\
Thyroidectomy & 4 & - \\
Carotid endarterectomy & 2 & - \\
Trauma & - & 16 \\
\hline
\end{tabular}

the literature are presented in Table 1. The lack of overlap in the categories (excepting "idiopathic") is striking and suggests that otolaryngologists are still approaching the topic with individual notions of causation rather than any consensus. In addition to these causes, vocal fold paresis has been reported to result from myasthenia gravis $[5,6]$, Charcot-Marie-Tooth neuropathy [7], spinocerebellar atrophy [8], implantation of vagal nerve stimulators [9, 10], thyroid disease [11], thymoma [12], and probably other neurologic insult or systemic disease.

\section{Incidence}

Some have argued that paresis of one or more laryngeal nerves, and in particular superior laryngeal nerve paralysis, is prevalent among patients with voice disorders; incidences as high as $80 \%$ have been proposed [13]. Were that approximately accurate, it would imply that the laryngeal nerves are uniquely fragile among cranial nerves; no other peripheral nerve has nearly as a high incidence of dysfunction. There is no physiologic or pathologic basis for belief that the larynx is handicapped in this way.

The available literature, although scant, offers a different picture. Heman-Ackah and Barr [14*0] identified 19 cases of vocal fold paresis (of 22 suspected cases) over 13 months. Koufman et al. [15••] identified 50 patients over 4 years in a similar setting. Merati et al. [16] published one of the few series to report paresis alongside paralysis; 29 cases of paresis were identified in 1 year, compared with 46 cases of paralysis. Another series restricted to superior laryngeal nerve paralysis-itself a contentious diagnosis - reported 71 cases of paresis and 55 cases of paralysis identified over 7 years [17]. These findings suggest that vocal fold paresis is not common, and appears to occur with about the same incidence as paralysis. When we take into account that each of these articles reports the experience of a specialized laryngology practice, vocal fold paresis is likely even less prevalent in the general population. As for so many other aspects of this disorder, more data are essential.

Prognosis

There is virtually no evidence regarding the clinical course of vocal fold paresis. Common sense suggests that it is probably analogous to that of paralysis; there is some potential for spontaneous improvement and even full recovery in the 6-12 months following onset. As the injury in incomplete, and neural conduits for regeneration are by definition preserved, this potential very likely exceeds that of paralysis. On the other hand, because of the relative mildness of the symptoms and the subtle examination findings, many patients tend to come for diagnosis relatively late in their clinical course, after the period for spontaneous recovery has elapsed.

\section{Clinical Presentation and Diagnosis}

\section{Symptoms}

As one would expect, symptoms of paresis are predominantly those of glottic insufficiency, even when both sides of the larynx are involved. This is because it is rare for paresis to be so dense that it impairs abduction to the extent that the airway is meaningfully narrowed. On the other hand, phonatory glottic function is affected by even mild deficits of neural input. Even when glottic closure appears grossly adequate, asymmetries in vocal fold tension may affect pitch, vocal stamina, and high-intensity or lowintensity phonation.

Hoarseness is the commonest complain of patients with vocal fold paresis $[15 \bullet \cdot, 18 \bullet \cdot$. In addition to rough voice quality, patients may complain of breathy voice, breathlessness during voicing, increased phonatory effort, difficulty in speaking over background noise or being heard at a distance, and fading of voice with use and pitch limitations [15••]. Symptoms may be present only in adverse acoustic environments-when teaching class, for example, or when speaking outdoors. Symptoms commonly become more pronounced over the course of the day, as compensatory mechanisms fatigue. As with idiopathic vocal fold paralysis, it is not unusual for symptoms to began after an upper respiratory tract infection. Associated swallowing symptoms are rare.

Although we concern ourselves predominantly with paresis of laryngeal motor nerves in this article, there is no reason to suppose that sensory paresis may not also exist, 
with distinct symptoms, either separately on concurrently. Means of laryngeal sensory testing, especially with respect to hypersensitivity or dysesthesias, remain rudimentary or absent, making description difficult. Globus sensation, chronic cough, and laryngospasm have all been attributed to laryngeal sensory neuropathy $[19,20 \bullet]$. For cough, at least, some success has been achieved via empiric treatment with neuromodulating medication [21], lending credence to such speculation. Laryngeal neuropathy has also been hypothesized to contribute to an entire spectrum of laryngeal movement abnormalities, including paradoxical vocal fold motion, paroxysmal vocal cord dysfunction, and so-called irritable larynx syndrome. Sorting among this poorly defined body of diagnoses and descriptions has been made challenging by important ambiguities and contradictions in the current terminology, and remains beyond the scope of this review.

\section{Signs: Laryngoscopy}

It is not much of an exaggeration to say that a determined observer may find signs of paresis in virtually every larynx. In contrast to systems such as the extraocular muscles in humans, where discoordination carries a functional disadvantage, it is likely that there is some evolutionary tolerance for asymmetry in vocal fold motion. So long as glottic closure is adequate and brisk, abduction or adduction lags probably have no impact on airway protection. Thus, much asymmetry in vocal motion is probably without clinical significance. Yet it may also be the only clinical sign of paresis.

The subject is usually discussed on the basis of expert opinion; only recently has it been subjected to critical study. As often happens in the absence of evidence, opinions are strongly held. In general, vocal fold hypomobility and glottic insufficiency are usually considered to be the commonest signs of motor paresis. In practice, such signs can be difficult to discern because of their subtlety, and even more difficult for practitioners to agree about. Height mismatch is perhaps the quintessential example of such a finding, often invoked but rarely with consensus. "Bowing" is a particularly imprecise, essentially descriptive term that refers to a concavity of the membranous vocal fold which may result from hypotonia or atrophy, the latter not necessarily neurogenic. It is sometimes even used as a diagnosis, but is probably best avoided altogether because of its ambiguity.

Fleischer et al. [22] have pointed out that observation of arytenoid motion may be misleading and have emphasized the importance of observing the vocal process and the membranous vocal fold itself. In contrast, Sufyan et al. [23•] have presented a well-reasoned argument in favor of observing arytenoid rotation as a sign of lateral cricoarytenoid paresis especially. Using a systematic approach for evaluation, their observations proved to have high interrater reliability. Rubin et al. [24] have described the use of repetitive phonatory tasks during examination to fatigue inadequately innervated musculature to accentuate vocal fold hypomobility. Carroll et al. [25•] have formally described a method used by many to estimate glottic insufficiency using frame-by-frame analysis of the glottal cycle as recorded on stroboscopy. Belafsky et al. [26•] have pointed out that supraglottic hyperfunction is a sign of underlying glottic insufficiency, and should by itself raise the question of paresis, especially when asymmetric. This feature is particularly useful in clinical practice because it is more distinct than many others.

Strobovideolaryngoscopy offers a means of assessing the characteristics of vocal fold vibration in addition to gross motion. Because these reflect differences in underlying vocal fold tension, they would be expected to be more sensitive to the presence of mild paresis. A case report suggests a similar potential for high-speed imaging [12]. Indeed, vibratory asymmetry-including differences in phase and amplitude-has been shown to correlate well with the presence electromyographic abnormalities, but expert judges fared poorly in their attempts to identify the side of the paresis on the basis of the asymmetry [27].

Superior laryngeal nerve paresis and paralysis is often discussed as a separate subgroup, probably with little justification given the established variability in laryngeal neuropathy, except in the case of surgical injury. It has been the subject of the sole in vivo experimental study in this area. Roy et al. [28] distributed examinations of subjects with lidocaine-induced and electromyographically verified superior laryngeal nerve palsies to blinded observers. The only reliable sign proved to be deviation of the petiole of the epiglottis to the side of the paresis during high-pitched phonation, a sign never before advocated by even the most opinionated practitioner, and certainly not used clinically. Its clinical utility awaits further study, and the work of Roy et al. reminds us to be circumspect in our assumptions, and perhaps above all to remain humble.

Before we leave the subject of laryngoscopy, it is worth remembering that paresis may be the cause of other pathologic findings in the larynx. Two groups have pointed out the relation between contact lesions and underlying glottic insufficiency from paresis [29, 30]. Koufman and Belafsky [31] have suggested that pseudocysts-fusiform, translucent lesions of the vibratory margin-are invariably a consequence of underlying paresis. The hypothesized mechanism is increased sheering trauma from effortful glottic closure. Although intriguing, this explanation remains problematic, as it does not easily account for the preponderance of these lesions in women, nor the nearuniversal unilaterality of the lesion (trauma for effortful 
glottic closure is presumably imparted bilaterally), nor the fact that most such lesions do not recur after surgical removal. Furthermore, even casual reflection reveals that such lesions are almost never seen in cases of frank paralysis, even when glottic closure is adequate, as after spontaneous improvement or medialization. These issues notwithstanding, the alert examiner may still use the presence of such lesions to direct his or her attention to the possibility of an underlying paresis.

\section{Electromyography}

Virtually every author to address the subject of paresis has remarked on the discrepancy between clinical observations and electromyographic findings $[14 \bullet \bullet, 15 \bullet, 24,32]$. In one series, about one in four patients had electromyographic findings not predicted by the endoscopic examiner [33•]; in another, the incidence of unexpected findings was higher still—about $40 \%$ [14.•]. The presence of innocent asymmetries in laryngeal motion, to which I alluded earlier, is no doubt a confounder here as well.

Often dismissed as being subjective, laryngeal electromyography can be less so than laryngoscopy and stroboscopy in the case of neural compromise. Findings of fibrillations, positive sharp waves, or polyphasic motor unit action potentials are clear and unambiguous signs of neurologic impairment, either new or old. Unfortunately, in paresis, such frankly abnormal findings are not always present or discernable against the signal of preserved muscle activity. There may be only decreased recruitment of otherwise normal appearing motor unit action potentials. Because this relative change can be small, and mimicked by incomplete muscle activation, or suboptimal needle placement, there remains a role for physician judgment and-inevitably — error. Another feature is perhaps of even greater significance: the maximal interference pattern in striated muscle is typically present at only $30 \%$ of maximal isometric contraction, leaving open the possibility that even fairly substantial paresis may be completely overlooked by electromyography. Thus, although its specificity for vocal fold paresis is probably high, its sensitivity is certainly less, and potentially much lower that we suspect. Although electromyography can provide information that laryngoscopy cannot, it is not clear that it is a more accurate tool than laryngoscopy or stroboscopy, and there may be little basis for regarding it as the gold standard for diagnosis of vocal fold paresis, as very many studies do.

\section{Treatment}

In published reports, patients with vocal fold paresis have been reported to respond to steroids and antiviral agents, voice therapy, injection augmentation, and unilateral or bilateral medialization laryngoplasty. Because the vocal fold retains substantial movement, procedures which require immobilization of the arytenoid, such as arytenoid adduction, are not useful. For similar reasons, reinnervation is poorly suited to the diagnosis, because even in cases of complete paralysis reinnervation cannot restore motion beyond the level to which it is usually preserved in paresis to begin with.

In light of the diagnostic difficulties examined herein, it is worth highlighting the use of treatment for diagnosis. The renewed availability of low-morbidity injection augmentation in the office, made possible by the advent of improved visibility from distal-chip endoscopes and more forgiving injectable materials, has made it possible to contemplate a treatment trial even in patients in whom the diagnosis is not certain $[34,35]$. Symptomatic improvement after injection augmentation validates the diagnosis more directly than either laryngoscopy or electromyography. Although it is perhaps less elegant than diagnosis by these two modalities, it also directly answers the very practical question: "Will augmentation help this patient's symptoms?" As a result, trial injection augmentation may be the most reliable and practical means of investigating the diagnosis of vocal fold paresis today.

\section{Conclusions}

Despite a sound pathophysiologic basis for the existence of paresis, interest in and acceptance of the diagnosis of paresis is relatively recent. Most aspects of this entityincluding prevalence, causative factors, natural history, laryngoscopic and stroboscopic signs, and relation to other diseases-continue to require clarification. There is good reason to suppose that paresis yields a clinical picture at least as heterogeneous as that of paralysis and a good deal subtler. Diagnosis remains the greatest challenge, as laryngoscopy does not reliably distinguish innocent laryngeal asymmetries from those due to paresis. Electromyography may be less reliable than initially appreciated, principally because of a lack of sensitivity. In this context, treatment trials may assume greater importance until diagnostic tools are refined.

The most encouraging aspect is that the means to help most patients with paresis already exists in the repertoire of interventions developed to treat paralysis. Its effective application, however, depends very much on diagnostic accuracy.

Disclosure Lucian Sulica declares no conflict of interest. 


\section{References}

Papers of particular interest, published recently, have been highlighted as:

- Of importance

•- Of major importance

1. Blitzer A, Jahn AF, Keidar A. Semon's law revisited: an electromyographic analysis of laryngeal synkinesis. Ann Otol Rhinol Laryngol. 1996;105:764-9.

2. - Bielamowicz S, Stager SV. Diagnosis of unilateral recurrent laryngeal nerve paralysis: laryngeal electromyography, subjective rating scales, acoustic and aerodynamic measures. Laryngoscope. 2006;116:359-64. This is a thorough multidimensional assessment revealing the heterogeneity of vocal fold paralysis.

3. Benjamin B. Vocal cord paralysis, synkinesis and vocal fold motion impairment. ANZ J Surg. 2003;73:784-6.

4. Badia PI, Hillel AT, Shah MD, Johns MJ, Klein AM. Computed tomography has low yield in the evaluation of idiopathic unilateral vocal fold paresis. Laryngoscope. 2013;123:204-7.

5. Teramoto K, Kuwabara M, Matsubara Y. Respiratory failure due to vocal cord paresis in myasthenia gravis. Respiration. 2002;69: 280-2.

6. Mao VH, Abaza M, Spiegel JR, Mandel S, Hawkshaw M, Heuer RJ, Sataloff RT. Laryngeal myasthenia gravis: report of 40 cases. J Voice. 2001;15:122-30.

7. Sulica L, Blitzer A, Lovelace RE, Kaufmann P. Vocal fold paresis of Charcot-Marie-Tooth disease. Ann Otol Rhinol Laryngol. 2001;110:1072-6.

8. Isozaki E, Naito R, Kanda T, Mizutani T, Hirai S. Different mechanism of vocal cord paralysis between spinocerebellar atrophy and multiple system atrophy. J Neurol Sci. 2002;197:37-43.

9. Shaw GY, Sechtem P, Searl J, Dowdy ES. Predictors of laryngeal complications in patients implanted with the Cyberonics vagal nerve stimulator. Ann Otol Rhinol Laryngol. 2006;115:260-7.

10. Zalvan C, Sulica L, Wolf S, Cohen J, Gonzales-Yanes O, Blitzer A. Laryngopharyngeal dysfunction from the implantable vagal nerve stimulator. Laryngoscope. 2003;113:221-5.

11. Heman-Ackah YD, Joglekar SS, Caroline M, Becker C, Kim EJ, Gupta R, Mandel SM, Sataloff RT. The prevalence of undiagnosed thyroid disease in patients with symptomatic vocal fold paresis. J Voice. 2011;25(4):496-500.

12. Mortensen M, Woo P. High-speed imaging used to detect vocal fold paresis: a case report. Ann Otol Rhinol Laryngol. 2008;117(9): 684-7.

13. Koufman JA. Oral presentation. Ninth biennial Phonosurgery Symposium, University of Wisconsin, 6-8 July 2006.

14. • Heman-Ackah YD, Barr A. Mild vocal fold paresis: understanding clinical presentation and electromyographic findings. J Voice. 2006;20:269-81. This is one of the large series of vocal fold paresis in the literature. The investigators provide a complete perspective.

15. • Koufman JA, Postma GN, Cummins MM, Blalock PD. Vocal fold paresis. Otolaryngology Head Neck Surg. 2000;122:537-41. This is another large series of vocal fold paresis in the literature. It is a complete review from a contrasting point of view.

16. Merati AL, Shemirami N, Smith TL, Toohill RJ. Changing trends in the nature of vocal fold motion impairment. Am J Otolaryngol. 2006;27:106-8.

17. Dursun G, Sataloff RT, Spiegel JR, Mandel S, Heuer RJ, Rosen DC. Superior laryngeal nerve paresis and paralysis. J Voice. 1996;10:206-11.
18. •• Simpson CB, Cheung EJ, Jackson CJ. Vocal fold paresis: clinical and electrophysiologic features in a tertiary laryngology practice. J Voice. 2009;23(3):396-8. This is another large and well-documented series, with specific discussion of electromyography.

19. Amin MR, Koufman JA. Vagal neuropathy after upper respiratory infection: a viral etiology? Am J Otolaryngol. 2001;22:251-6.

20. - Rees CJ, Henderson AH, Belafsky PJ. Postviral sensory neuropathy. Ann Otol Rhinol Laryngol. 2009;118(4):247-52. This provides a well-reasoned and clinically supported description of laryngeal sensory neuropathy.

21. Lee P, Woo P. Chronic cough as a sign of laryngeal sensory neuropathy: diagnosis and treatment. Ann Otol Rhinol Laryngol. 2005;114:253-7.

22. Fleischer S, Schade G, Hess MM. Office-based laryngoscopic observations of recurrent nerve paresis and paralysis. Ann Otol Rhinol Laryngol. 2005;114(6):488-93.

23. - Sufyan AS, Kincaid JC, Wannemuehler TJ, Halum SL. The interarytenoid spatial relationship: accuracy and interrrater reliability for determining sidedness in cases of unilateral adductor paresis. J Voice. 2013;27(1):90-4. This provides an interesting and clinically useful anatomical insight into laryngoscopic observations.

24. Rubin AD, Praneetvatakul V, Heman-Ackah Y, Moyer CA, Mandel S, Sataloff RT. Repetitive phonatory tasks for identifying vocal fold paresis. J Voice. 2005;19:679-86.

25. - Carroll TL, Wu YHE, McRay M, Gherson S. Frame by frame analysis of glottic insufficiency using laryngovideostroboscopy. J Voice. 2012;26(2):220-5. This describes a commonly used estimate of glottic insufficiency.

26. • Belafsky PC, Postma GN, Reulbach TR, Holland BW, Koufman JA. Muscle tension dysphonia as a sign of underlying glottal insufficiency. Otolaryngol Head Neck Surg. 2002;127:448-51. This describes the tremendously useful clinical sign of glottic insufficiency.

27. Simpson CB, May LS, Green JK, Eller RL, Jackson CE. Vibratory asymmetry in mobile vocal folds: is it predictive of vocal fold paresis? Ann Otol Rhinol Laryngol. 2011;120(4):239-42.

28. Roy N, Smith M, Houtz D. Laryngoscopic features of external superior laryngeal nerve denervation: revisiting a century-old controversy. Ann Otol Rhinol Laryngol. 2011;120:1-8.

29. Carroll TL, Gartner-Schmidt J, Statham MM, Rosen CA. Vocal process granuloma and glottal insufficiency: an overlooked etiology? Laryngoscope. 2010;120:114-20.

30. Halum SL, Miller P, Early K. Laryngeal granulomas associated with superior laryngeal nerve paresis. J Voice. 2010;24(4):490-3.

31. Koufman JA, Belafsky PC. Unilateral or localized Reinke's edema (pseudocyst) as a manifestation of vocal fold paresis: the paresis podule. Laryngoscope. 2001;111:576-80.

32. Altman KW. Laryngeal asymmetry on indirect laryngoscopy in a symptomatic patient should be evaluated with electromyography. Arch Otolaryngol Head Neck Surg. 2005;131:356-60.

33. - Koufman JA, Postma GN, Whang CS, Rees CJ, Amin MR, Belafsky PC, Johnson PE, Connolly KM, Walker FO. Diagnostic laryngeal electromyography: The Wake Forest experience 1995-1999. Otolaryngol Head Neck Surg. 2001;124:603-6. This is a valuable review of the clinical utility of electromyography in laryngology. Note the high incidence of unexpected findings.

34. Sulica L, Rosen CA, Postma GN, Simpson B, Amin M, Courey M, Merati A. Current practice in injection augmentation of the vocal folds: indications, treatment principles, techniques and complications. Laryngoscope. 2010;120:319-25.

35. Carroll TL, Rosen CA. Trial vocal fold injection. J Voice. 2010;24(4):494-8 